\title{
Las ideas de Felipe Picatoste sobre el vocabulario matemático en la undécima edición del diccionario de la Real Academia Española*
}

\author{
FRANCISCO JAVIER SÁNCHEZ MARTÍN \\ Universidad de Murcia
}

\section{INTRODUCCIÓN}

El trabajo de Felipe Picatoste y Rodríguez (Madrid, 1834-1892) ha girado, como reconoce en el prólogo de El tecnicismo matemático, en torno al vocabulario matemático. ${ }^{1}$ En este sentido, su continua revisión del Diccionario de la lengua castellana le llevó a percatarse de los errores e inexactitudes del mismo, lo que le animó a componer finalmente su obra, guiado por el consejo del académico de número Antonio Ferrer del Río (1814-1872), bibliotecario de la Real Academia Española entre 1867 y 1872 y miembro de la Sociedad Económica matritense. ${ }^{2}$

El tecnicismo matemático, redactado con el deseo de que «algún Académico curioso, que le lea, tenga presente algunas de sus observaciones» (Picatoste, 1873: 5), está dividido en seis partes: «Las academias ante la ciencia», «De la falta de voces técnicas en el diccionario», «De la falta de exactitud en las definiciones», «Catálogo de voces matemáticas que faltan en el diccionario», «Catálogo de voces matemáticas mal definidas o explicadas en el diccionario», «Sinónimos matemáticos».

En las tres primeras partes el autor desarrolla las ideas que le sirven de aparato teórico para sostener las restantes en las que cataloga las voces matemáticas objeto de

* Esta investigación se ha realizado merced al proyecto Diccionario histórico de la minería: prolegómenos (11845/PHCS/09), financiado por la Fundación Séneca, y se integra en el marco del proyecto FFI2010-16324/FILO, financiado por la Dirección General de Investigación.

${ }^{1}$ Escribió importantes obras, entre ellas, Apuntes para una biblioteca científica española del siglo XVI (1891), Explicación del nuevo sistema legal de pesas y medidas (1853), Vocabulario matemáticoetimológico (1862), Principios y ejercicios de aritmética y geometría (1865), Elementos de matemáticas (1870), Los diálogos del bachiller Juan Pérez de Moya (1875) o Elementos de física y química (1889) (vid. Enciclopedia universal ilustrada europeo-americana, 1975: s. v. Picatoste, Felipe).

${ }^{2}$ Ferrer del Río ingresó en la Academia el 29 de mayo de 1853, ocupando la silla Q, en sustitución de Juan Nicasio Gallego. Formado en lenguas modernas y clásicas, matemáticas y taquigrafía, fue periodista notable y llegó a dirigir varios periódicos madrileños. Su producción fue muy variada; pueden destacarse, entre otras, las numerosas biografías de ilustres escritores (Zamora Vicente, 1999: 195-196). 
estudio. Nuestro propósito es detenernos en las observaciones, reflexiones e ideas programáticas que Picatoste despliega sobre el lenguaje científico y los tecnicismos matemáticos.

\section{Los TECNICISMOS Y LA REAL ACADEMIA ESPAÑOLA}

La aparición de la undécima edición del diccionario de la Academia Española venía a suponer una renovación para el diccionario de lengua ${ }^{3}$. El sentir de las novedades que la nueva edición deparaba al público era unánime en todos los círculos sociales de la época. Sin embargo, el primer acercamiento a la parte científica del mismo defraudó a Picatoste al percibir lo que era una opinión unánime: la falta de atención a las voces técnicas, hecho que a la postre se convirtió en el centro de las críticas que la Corporación iría recibiendo. Picatoste da cabida al desencuentro existente en este terreno:

Nos lamentamos de que la Academia no haya hecho un esfuerzo para conformar el lenguaje científico con el castizo; para que en el Diccionario se refleje de algun modo el progreso de la ciencia [...] y para evitar en lo posible que los Académicos se lamenten de que los hombres de ciencia «les destrozan el lenguaje», y estos á su vez, desde las mismas cátedras, pugnen por romper el yugo de la Academia, y proclamen que con el Diccionario en la mano no pueden hablar científicamente ni aun los mismos Académicos (Picatoste, 1873: 8).

El desarrollo cultural, el avance de las ciencias y técnicas y de las actividades en distintas parcelas propicia que el vocabulario científico engrose el lenguaje que con ellas se relaciona. La ciencia y sus investigadores tropiezan en su camino, según el autor, con «institutos cuyo lema es la invariabilidad, la tradición, el sosiego y quizá la inactividad». En este punto, Picatoste examina la confrontación de razones entre los hombres de ciencia y los académicos. Con respecto a los últimos, destaca el rechazo a las novedades léxicas y la sanción de la tradición, reflejada en el acopio de voces antiguas en el diccionario. Por parte de los otros, los científicos, critica el ideal de que el repertorio académico extienda sus límites para convertirse en un libro científíco. Con buen juicio, Picatoste se aviene a la tesis de que la obra académica no puede albergar voces sin reconocer si están bien o mal usadas, o si son innecesarias, a la vez que condenar al olvido las voces anticuadas por no servir para los nuevos procedimientos.

La posición ecléctica define el modo de pensar de Picatoste. Ni un extremo ni el otro. Así, el matemático defiende para el diccionario de la lengua, por un lado, la tradi-

\footnotetext{
${ }^{3}$ En su preámbulo al lector, la edición de 1869 anuncia: «La Academia Española fiel á su instituto, se ha aplicado sin descanso á revisar y perfeccionar esta su más importante y delicada obra. Atenta á las observaciones que se le han dirigido [...] ha seguido en la presente edición de su Diccionario el movimiento progresivo que en todo idioma necesariamente se verifica; pero sus pasos han sido lentos y mesurados, que no de otra suerte se conserva la integridad de las lenguas cultas y se asegura el acierto en su natural desenvolvimiento». Y se añade: «Sale esta edición notablemente aumentada con algunos centenares de vocablos, de frases y de nuevas acepciones» (DRAE-1869).
} 
ción, el rico caudal léxico antiguo y, por otro, las innovaciones léxicas que el progreso científico impone. Tradición y modernidad, línea fina sobre la que la Academia debe moverse para no verse arrinconada. Para Picatoste la docta Corporación debe estar a la cabeza del movimiento incesante de la sociedad moderna empleando dos de sus poderosas armas: «con la imposición de la autoridad y el prestigio que da el valor» (Picatoste, 1873: 12).

\section{EL DICCIONARIO ACADÉMICO Y LAS VOCES DE CIENCIA}

Con respecto a la recepción de voces en el diccionario, Picatoste adopta los mismos criterios que la Academia mantiene, por más que ésta, en su opinión, los haya incumplido. La edición consultada por el matemático es la vigente en el momento en que elabora su obra, la undécima edición (1869). Esta edición presenta entre sus novedades la supresión de las equivalencias latinas mantenidas desde Autoridades.

Además, los preceptos que guían la labor de los académicos se resumen, primero, en el rechazo al aumento indiscriminado de vocablos y, a la vez, la sanción de nuevas palabras indispensables «de recta formación, é incorporadas en el Castellano por el uso de las personas doctas» (DRAE-1869), y segundo en la rehabilitación de voces para lo que se elimina la calificación de anticuadas que muchas de ellas llevaban.

Pese a ello, Picatoste cree que la Academia desoye sus propias reglas por cuanto rechaza en su obra términos que, precisamente, deberían contar con su aval por poseer dos cualidades esenciales para los académicos: el marchamo de la antigüedad y del uso.

Por eso no se explica el que términos del álgebra, geometría, geometría analítica o del cálculo no estén presentes en la nomenclatura del diccionario. Retóricamente sentencia: «Ignoramos si en la Academia hay alguno que haya sido profesor de matemáticas; pero seguramente la gran mayoría de sus ilustrados individuos habrá cursado, á lo ménos, un año de estas ciencias» o «¿Podria tampoco algun Académico leer con fruto obras antiguas de matemáticas en lengua castellana sin saber lo que es abaco, almucabala, aporeo, helmuayo, isógono, [...] y otras muchas que tampoco se encuentran en el diccionario?» (Picatoste, 1873: 15-16).

Efectivamente, son voces que provienen de la antigüedad clásica, proceden del griego y del latín, las emplearon Apolonio y Euclides, o se acuñaron bajo la dominación de los árabes ${ }^{4}$, por ende, vienen empleándose desde hace siglos en matemáticas. Son por consiguiente de ascendencia ilustre, no como las tomadas de las lenguas vivas,

\footnotetext{
${ }^{4}$ «Los árabes introducen en Europa las cifras índicas; cultivan la Aritmética y la Geometría; traducen y esparcen en el mundo cristiano los libros griegos; perfeccionan, y según algunos historiadores crean el Álgebra o Almucabala y la Trigonometría; y fundan por do quiera escuelas, academias y bibliotecas, que son brillantes faros de purísima luz en aquellos siglos medios de profundas y densas tinieblas» (Echegaray, 2007: 14).
} 
«que crispan los nérvios a los académicos poco amigos de las novedades, ofenden los oidos de los puristas, y pueden ser rechazadas por algun matemático amante de la lengua pátria» (Picatoste, 1873: 16).

De ahí que proponga una regla universal: «La inclusión de todas las palabras, sin cuyo constante y frecuente uso no fuera posible explicar una rama de la ciencia» (Picatoste, 1873: 14).

La omisión de estos términos matemáticos con abolengo representa, a juicio del autor, el olvido que sufren las ciencias exactas - «que son una serie de principios evidentes y demostrables»—-, al contrario de lo que sucede en otros ámbitos terminológicos como la retórica o la medicina. Por ello una tarea apremiante es la incorporación de estos tecnicismos y la reforma de la definición de otros tantos, ya que «en matemáticas, el Diccionario no ha llegado siquiera al febril siglo pasado; se encuentra en el siglo XVII» (Picatoste, 1873: 18), según él mismo confiesa.

\section{CONSIDERACIONES EN TORNO AL LENGUAJE CIENTÍFICO}

Las primeras ideas que sobre el lenguaje científico plasma Picatoste en El tecnicismo matemático tienen que ver con las palabras usuales del lenguaje corriente, el «puro y castizo de que es guardadora la Academia». Ese léxico no puede ostentar la condición de lenguaje científico, sino que, en su opinión, el lenguaje científico es aquel que surge de la actividad inventora y de la comunicación entre especialistas: «Está sujeto al movimiento progresivo, al oleaje incesante y á la perpétua discusion de teorías, aplicaciones y objetos nuevos con que cada dia se enríquece el caudal de la ciencia» (Picatoste, 1873: 20).

Por tanto, ¿cuáles son las características que exhibe el vocabulario que ostenta la condición de científico?:

Es un lenguaje limitado, especial, que exige un rigor y una propiedad. [...] Es más constante en sus giros y en sus construcciones, más pobre en sus formas; pero más delicado en el uso de las palabras y en el rigor de los conceptos. Aunque sujeto á las variaciones del idioma, y á la invasión de voces nuevas y extranjeras, más que otro alguno, su aridez y concision rechazan la variedad (Picatoste, 1873: 20).

Empleando términos más actuales el lenguaje científico se caracteriza por su carácter limitado, neutralidad, fijación, simpleza en el empleo de estructuras, concisión, precisión y univocidad; rasgos y cualidades que siguen utilizándose en la descripción de los registros de especialidad (vid. Cabré, 1993 y 2003 y Gutiérrez Rodilla, 1998). Además, es un lenguaje en el que predominan las innovaciones léxicas, esto es, los neologismos y los préstamos, que, indudablemente, entran por la vía de «la renovación de las técnicas» (Fernández Sevilla, 1974: 138).

Por otra parte, cabe preguntarse por los términos que deben tener cabida en el diccionario de lengua. Aunque Picatoste no defiende la aspiración de que el diccionario se convierta en un texto científico, a la vez ser un manual de matemáticas, medicina, química, etc., sí aspira a que el repertorio lexicográfico contenga el rigor necesario - 
ofrezca para las voces una explicación clara, exacta y útil- y contemple los términos matemáticos que él propone. Las palabras del lenguaje matemático pueden agruparse en los siguientes tres grupos:

1) Términos exclusivamente técnicos, esto es, consagrados por la tradición y sin uso fuera del ámbito científico. Voces pertenecientes a los principios y fundamentos de las matemáticas o las propiedades de la cantidad y de las figuras: cateto, cicloide, cisoide, hipotenusa, isógono, etc.

2) Palabras del lenguaje común.

3) Voces formadas etimológicamente del latín y del griego, como las referidas al nuevo sistema métrico decimal o a los nombres de las curvas de grados superiores.

Con respecto al segundo grupo, en su ideario el lenguaje común participa en la expresión de los conceptos científicos. Reconoce explícitamente la posibilidad de que ciertas palabras del léxico ordinario pasen a funcionar como tecnicismos en un momento dado. Se acerca, por tanto, a la concepción de los estudios actuales que muestran la importancia del léxico común en la nomenclatura científica (cf. Gili Gaya, 1964; Fernández Sevilla, 1974; Cabré, 1993; Gutiérrez Rodilla, 1998). Contempla para ello diversos procesos como el de neología sintáctica: «Limitando unas veces su extensión, variando algun tanto otras su primitivo significado, ya convirtiendo los adjetivos en sustantivos, ya formando nuevos adjetivos para distinguir entre sí propiedades» (Picatoste, 1873: 22).

\section{LA TERMINOLOGÍA MATEMÁTICA EN EL DICCIONARIO ACADÉMICO DE 1884}

En el siglo XIX la mayoría de las críticas hacia la Academia se centraban en el escaso número de entradas contenidas en su diccionario y en la poca atención hacia neologismos y tecnicismos. Por ello Picatoste incorpora un catálogo de voces donde reúne una nomenclatura formada por 212 voces que la Academia se negaba a recoger, «rechazándolas porque no forman parte del lenguaje usual, por su origen, por su especialidad, o por cualquier otra razon que nunca faltaria a la sutileza e ingenio de los Académicos» (Picatoste, 1873: 19).

En primer lugar, recopilaremos este conjunto léxico y realizaremos una clasificación taxonómica del mismo. En segundo lugar, comprobaremos si los tecnicismos matemáticos que no se encuentran lematizados en la edición de 1869, que Picatoste manejó, se incorporaron en la edición siguiente; lo que nos permitirá comprobar si efectivamente se cumplen las expectativas que la edición de 1884 despertó respecto al aumento considerable de palabras técnicas. Finalmente, analizaremos las voces incorporadas por Picatoste en su catálogo con el objeto de examinar las observaciones y matización subjetivas que introduce en alguna de ellas.

Para la clasificación conceptual de las más de doscientas voces matemáticas propuestas por Felipe Picatoste hemos considerado las siguientes disciplinas, según el 
ámbito de especialidad al que pueden adscribirse: aritmética, álgebra, geometría, trigonometría y otras voces matemáticas.

Aritmética: ábaco, algoritmia, antilogaritmo, aritmología, aritmógrafo, aritmómetro, canon, característica, congruencia, conjugado, conjunta, dactilonomia, digital, dignidad, discreta, efección, elemento, equimúltiplo, figurado, fluente, fluxión, generador, integral, identidad, igual, igualdad, (número) incomplejo, indeterminado, interpolar, irregular, ley, límite, logarítmica, logística, medial, menos, módulo, (número) perfecto, (número) primo, punto, dos puntos, rabdología, residuo, resta, resto, signo, sordo, submúltiplo, subtriple, sumatorio, superposición, variable.

Álgebra: aditivo, almucábala, bicuadrado, bimedial, combinación, cuatrinomio, derivada, despejar, destruir, determinado/determinar, dimensión, discusión, divergente, eliminación, eminencial, exégesis, exponencial, factorial, fórmula, función, grado, grupo, homogéneo, índice, isomería, multinomio, ordenatriz, recurrente, reducida, transposición.

Geometría: abscisa, acidoides, ambígena, ambligonio/amblígono, aplicada, apomecometría, aporeo, apotema, baculametría, bicóncavo, biconvexo, bisecante, bisecar, bisector, braquistocrona, broqueas, casinoide, casquete, cateto, ciligonia, cisoide, coincidencia, complemento, concoide, construcción, contorno, conversión, coordenada, corona, cultelación, diedro, directriz, eje, elipsoide, eliptoides, epicicloide, epipedometría, equiangulación, equiangular, equidistancia, equidistante, equinomias, equivalencia, espíricas, flecha, generatriz, helicoide, helmarise ${ }^{\mathbf{5}}$, helmuayo, hiperboloide, hoja, homocéntrico, horometría, huso, icnografía, idéntico, isógono, isoperímetro, kiliógono, lado, lateral, lemniscata, longimetría, lugar, lúnula, metrología, normal, oblicuángulo, odómetro, ordenada, ortogonal, ortogonio, osculación, osculador, oxígono/oxigonio, pantogonia, paraboloide, paracéntrico, pelecoide, piramidoide, planimetría, poligonometría, porisma, proyección, proyectante, radial, rectificar, regular, resecta, semejante, simetría, solidez, subcontrarios, suplemento, tautocrona, teodolito, tiralíneas, traza, triangulación, tridente, triedro, trilátero, trocoide, variación, vector, zetema, zona.

Trigonometría: cosecante, coseno, cotangente, subnormal, subtangente, tractriz, transcendente.

Otras voces matemáticas: aorista, apótome, asimetría, constante, cuatrillón, diferencial, diferenciar, imaginario, incremento, integración, integral, integrar, kiliada, miriárea, mirialitro, ochavillo, ordenar.

En la siguiente tabla seleccionamos los términos catalogados por Picatoste que estaban ausentes en la edición de 1869 del Diccionario de la lengua castellana. Hemos procedido a comprobar si éstos habían sido incorporados en la siguiente edición del diccionario académico. Los resultados obtenidos muestran, por una parte, que 96 de al-munharif.

${ }^{5}$ Posiblemente se trate de una errata de imprenta por helmuarife, del ár. helmuarif, calco 
términos, un 45\%, no aparecen lematizados en la edición de 1884. De los restantes 116 términos, 61 de ellos - representan un $29 \%$ - se incorporan a la nomenclatura del diccionario académico en su sentido matemático, mientras que son 55 (26\%) las voces que se recogen, pero no en la acepción matemática. Los términos que entran en este último grupo aparecen señalados en la tabla con la marca $\varnothing$.

En efecto, la duodécima edición del $D R A E$ supuso un cambio radical (Garriga 2001), incentivado sobremanera por las presiones y críticas ejercidas alrededor de la Academia, de modo que en sus Advertencias se declara ya el «considerable aumento de las palabras técnicas con que se la ha enriquecido», aunque con condicionantes explícitos como que los términos ofrecieran «señales inequívocas de duración» y pertenecieran a las ciencias y las artes de más general aplicación, además de «estar bien formados o ser de ilustre abolengo, como nacidos del griego o del latín» (DRAE-1884: Advertencias). Pese a lo limitado de la muestra seleccionada, puede afirmarse, sin embargo, que las innovaciones en el ámbito de las matemáticas se reducen a un 29 por ciento. Es mayoritario, por tanto, el número de tecnicismos que la Academia considera que no debe integrarse en su repertorio.

\begin{tabular}{|l|l|}
\hline & \multicolumn{1}{|c|}{ DRAE-1884 } \\
\hline ábaco & $\begin{array}{l}\text { Cuadro de madera con diez cuerdas o alambres paralelos y en cada uno } \\
\text { de ellos otras tantas bolas movibles, usado en las escuelas para enseñar } \\
\text { a los niños los rudimentos de la aritmética. }\end{array}$ \\
\hline abscisa & $\begin{array}{l}\text { Geom. Aplícase a la línea coordenada de que se hacen depender los } \\
\text { valores de las demás. } \| \text { Geom. Parte del eje o del diámetro de una curva, } \\
\text { comprendida entre su vértice y la ordenada correspondiente. }\end{array}$ \\
\hline acidoides & $\varnothing$ \\
\hline aditivo & \\
\hline algoritmia & \\
\hline almucábala & \\
\hline ambígena & Geom. Triángulo ambligonio \\
\hline $\begin{array}{l}\text { ambligonio/ } \\
\text { amblígono }\end{array}$ & \\
\hline antilogaritmo & \\
\hline aorista & \\
\hline aplicada & \\
\hline apomecometría & \\
\hline aporeo & \\
\hline apotema & \\
\hline
\end{tabular}




\begin{tabular}{|c|c|}
\hline apótome & \\
\hline aritmógrafo & \\
\hline aritmología & \\
\hline aritmómetro & \\
\hline asimetría & \\
\hline baculametría & \\
\hline bicóncavo & \\
\hline biconvexo & \\
\hline bicuadrado & \\
\hline bimedial & \\
\hline bisecante & \\
\hline bisecar & \\
\hline bisector & $\begin{array}{l}\text { Geom. Que divide una cosa en dos partes iguales. Aplícase comúnmente } \\
\text { a un plano o a una recta. }\end{array}$ \\
\hline braquistocrona & \\
\hline broqueas & \\
\hline canon & $\varnothing$ \\
\hline característica & $\begin{array}{l}\text { Mat. En los logaritmos, primero o primeros guarismos, anteriores a la } \\
\text { coma que los divide de sus respectivas fracciones decimales. La carac- } \\
\text { terística expresa números enteros. } \| \text { Mat. La misma coma (,) arriba } \\
\text { mencionada. }\end{array}$ \\
\hline casinoide & \\
\hline casquete & $\begin{array}{l}\text { Casquete esférico. Geom. Parte de la superficie de la esfera, cortada por } \\
\text { un plano que no pasa por su centro. }\end{array}$ \\
\hline cateto & $\begin{array}{l}\text { Geom. Cada uno de los dos lados que forman el ángulo recto en el } \\
\text { triángulo rectángulo. }\end{array}$ \\
\hline ciligonia & \\
\hline cisoide & \\
\hline coincidencia & $\varnothing$ \\
\hline combinación & $\varnothing$ \\
\hline complemento & Geom. Ángulo que falta a otro para completar uno recto. \\
\hline concoide & \\
\hline congruencia & $\varnothing$ \\
\hline conjugado & $\varnothing$ \\
\hline conjunta & $\varnothing$ \\
\hline
\end{tabular}




\begin{tabular}{|c|c|}
\hline constante & $\varnothing$ \\
\hline construcción & $\varnothing$ \\
\hline contorno & $\varnothing$ \\
\hline conversión & $\varnothing$ \\
\hline coordenada & $\begin{array}{l}\text { Geom. Aplícase a las líneas que sirven para determinar la posición de un } \\
\text { punto, y a los ejes o planos a que se refieren aquellas líneas }\end{array}$ \\
\hline corona & $\begin{array}{l}\text { Geom. Superficie comprendida entre dos circunferencias concéntricas } \\
\text { situadas en un plano. }\end{array}$ \\
\hline cosecante & Trig. Secante del complemento de un ángulo o de un arco. \\
\hline coseno & Trig. Seno del complemento de un ángulo o de un arco. \\
\hline cotangente & Trig. Tangente del complemento de un ángulo o de un arco. \\
\hline \multicolumn{2}{|l|}{ cuatrillón } \\
\hline \multicolumn{2}{|l|}{ cuatrinomio } \\
\hline \multicolumn{2}{|l|}{ cultelación } \\
\hline \multicolumn{2}{|l|}{ dactilonomia } \\
\hline derivada & $\varnothing$ \\
\hline despejar & $\begin{array}{l}\text { Álg. Separar por medio del cálculo una incógnita de las otras cantidades } \\
\text { que la acompañan en una ecuación. }\end{array}$ \\
\hline destruir & $\varnothing$ \\
\hline $\begin{array}{l}\text { determinado/ } \\
\text { determinar }\end{array}$ & $\varnothing$ \\
\hline diedro & Geom. Ángulo diedro. \\
\hline diferencial & $\begin{array}{l}\text { Mat. Aplícase a la cantidad infinitamente pequeña. \|Mat. V. Cálculo } \\
\text { diferencial. \| Mat. Diferencia infinitamente pequeña de una variable } \\
\text { determinada. }\end{array}$ \\
\hline diferenciar & $\varnothing$ \\
\hline digital & $\varnothing$ \\
\hline dignidad & $\varnothing$ \\
\hline dimensión & $\varnothing$ \\
\hline directriz & $\begin{array}{l}\text { Geom. Terminación f. de Director, } 2^{\mathrm{a}} \text { acep. [Geom. Dícese de la línea o } \\
\text { figura que determina el movimiento y condiciones de generación de } \\
\text { otra. En esta acep. La terminación f. es Directriz] }\end{array}$ \\
\hline discreta & $\begin{array}{l}\text { Mat. Aplícase a los números y cantidades que, a diferencia de los con- } \\
\text { cretos, constan de unidades no ligadas entre sí, aunque homogéneas. } \\
\text { Así, veinte hombres o diez árboles son cantidad discreta, y seis leguas } \\
\text { de camino o cinco brazas de cuerda lo son concreta. }\end{array}$ \\
\hline
\end{tabular}




\begin{tabular}{|c|c|}
\hline discusión & $\varnothing$ \\
\hline divergente & $\varnothing$ \\
\hline \multicolumn{2}{|l|}{ dos puntos } \\
\hline \multicolumn{2}{|l|}{ efección } \\
\hline eje & $\begin{array}{l}\text { Geom. Línea recta, real o imaginaria, que pasa por el centro de un sólido } \\
\text { de revolución, o de una curva plana, y en torno de la cual giran o podr- } \\
\text { ían girar así la curva como el cuerpo. }\end{array}$ \\
\hline elemento & $\varnothing$ \\
\hline \multicolumn{2}{|l|}{ eliminación } \\
\hline elipsoide & $\begin{array}{l}\text { Geom. Sólido formado por la revolución de una elipse sobre uno de sus } \\
\text { dos ejes. }\end{array}$ \\
\hline \multicolumn{2}{|l|}{ eliptoides } \\
\hline eminencial & $\varnothing$ \\
\hline \multicolumn{2}{|l|}{ epicicloide } \\
\hline \multicolumn{2}{|l|}{ epipedometría } \\
\hline \multicolumn{2}{|l|}{ equiangulación } \\
\hline \multicolumn{2}{|l|}{ equiangular } \\
\hline \multicolumn{2}{|l|}{ equidistancia } \\
\hline equidistante & Que equidista. \\
\hline \multicolumn{2}{|l|}{ equimúltiplo } \\
\hline \multicolumn{2}{|l|}{ equinomias } \\
\hline equivalencia & $\begin{array}{l}\text { Geom. Igualdad de áreas en dos figuras planas de distintas formas, co- } \\
\text { mo un triángulo y un cuadrilátero; y la de volúmenes en dos sólidos, } \\
\text { también de diversas formas, como una pirámide y un exaedro. }\end{array}$ \\
\hline \multicolumn{2}{|l|}{ espíricas } \\
\hline exégesis & $\varnothing$ \\
\hline \multicolumn{2}{|l|}{ exponencial } \\
\hline \multicolumn{2}{|l|}{ factorial } \\
\hline figurado & $\varnothing$ \\
\hline flecha & Geom. Sagita. \\
\hline fluente & $\varnothing$ \\
\hline fluxión & $\varnothing$ \\
\hline fórmula & $\begin{array}{l}\text { Mat. Resultado de un cálculo, cuya expresión, reducida a sus más sim- } \\
\text { ples términos, sirve de pauta y regla para la resolución de todos los } \\
\text { casos análogos. }\end{array}$ \\
\hline función & Mat. Cantidad cuyo valor depende del de otra u otras cantidades. \\
\hline
\end{tabular}




\begin{tabular}{|c|c|}
\hline generador & $\begin{array}{l}\text { Geom. Dícese de la línea o de la figura que por su movimiento engen- } \\
\text { dran respectivamente una figura o un sólido geométrico. En esta acep- } \\
\text { ción f. es Generatriz. }\end{array}$ \\
\hline generatriz & Geom. Generadora. \\
\hline grado & $\begin{array}{l}\text { Álg. Número de orden que expresa el de factores de la misma especie } \\
\text { que entran en un término. } \| \text { Álg. En una ecuación, el del término que lo } \\
\text { tiene mayor. } \| \text { Geom. Cada una de las partes iguales, que suelen ser } 360 \text {, } \\
\text { en que se considera dividida la circunferencia del círculo. }\end{array}$ \\
\hline grupo & $\varnothing$ \\
\hline \multicolumn{2}{|l|}{ helicoide } \\
\hline \multicolumn{2}{|l|}{ helmarise } \\
\hline \multicolumn{2}{|l|}{ helmuayo } \\
\hline \multicolumn{2}{|l|}{ hiperboloide } \\
\hline hoja & $\varnothing$ \\
\hline \multicolumn{2}{|l|}{ homocéntrico } \\
\hline homogéneo & $\varnothing$ \\
\hline \multicolumn{2}{|l|}{ horometria } \\
\hline huso & $\varnothing$ \\
\hline icnografia & $\varnothing$ \\
\hline idéntico & $\varnothing$ \\
\hline identidad & $\varnothing$ \\
\hline igual & $\begin{array}{l}\text { Mat. Signo de la igualdad formado de dos rayas horizontales y paralelas } \\
(=) \text {. }\end{array}$ \\
\hline igualdad & Mat. Identidad de valor entre dos o más cantidades. || Mat. Ecuación. \\
\hline imaginario & Mat. Cantidad imaginaria. \\
\hline \multicolumn{2}{|l|}{ incomplejo } \\
\hline incremento & $\varnothing$ \\
\hline indeterminado & $\varnothing$ \\
\hline indice & $\varnothing$ \\
\hline \multicolumn{2}{|l|}{ integración } \\
\hline integral & Mat. Cálculo integral. \\
\hline integrar & $\begin{array}{l}\text { Mat. Determinar una o más variables, conocidas sus diferencias infini- } \\
\text { tamente pequeñas, en virtud del cálculo integral. }\end{array}$ \\
\hline interpolar & $\varnothing$ \\
\hline
\end{tabular}




\begin{tabular}{|c|c|}
\hline irregular & $\varnothing$ \\
\hline isógono & $\varnothing$ \\
\hline \multicolumn{2}{|l|}{ isomería } \\
\hline \multicolumn{2}{|l|}{ isoperímetro } \\
\hline \multicolumn{2}{|l|}{ kiliada } \\
\hline \multicolumn{2}{|l|}{ kiliógono } \\
\hline lado & $\varnothing$ \\
\hline lateral & $\varnothing$ \\
\hline \multicolumn{2}{|l|}{ lemniscata } \\
\hline ley & $\varnothing$ \\
\hline límite & $\begin{array}{l}\text { Álg. Cada una de las dos cantidades máxima y mínima entre las cuales } \\
\text { se encuentran los valores de las raíces de una ecuación. }\end{array}$ \\
\hline logarítmica & $\varnothing$ \\
\hline \multicolumn{2}{|l|}{ logística } \\
\hline \multicolumn{2}{|l|}{ longimetría } \\
\hline lugar & $\varnothing$ \\
\hline lúnula & $\begin{array}{l}\text { Geom. Figura compuesta de dos arcos de círculo que se cortan volvien- } \\
\text { do la concavidad hacia el mismo lado. }\end{array}$ \\
\hline \multicolumn{2}{|l|}{ medial } \\
\hline menos & $\begin{array}{l}\text { Álg. y Arit. Signo de sustracción o resta que se representa por una rayita } \\
\text { horizontal (-). }\end{array}$ \\
\hline \multicolumn{2}{|l|}{ metrología } \\
\hline \multicolumn{2}{|l|}{ miriárea } \\
\hline \multicolumn{2}{|l|}{ mirialitro } \\
\hline módulo & $\varnothing$ \\
\hline \multicolumn{2}{|l|}{ multinomio } \\
\hline normal & $\begin{array}{l}\text { Geom. Aplícase a la línea recta o al plano perpendiculares a la tangente } \\
\text { en el punto de contacto. }\end{array}$ \\
\hline \multicolumn{2}{|l|}{ perfecto } \\
\hline primo & $\begin{array}{l}\text { Número primero o primo. Arit. El que sólo es exactamente divisible por } \\
\text { sí mismo o por la unidad, como } 5,7 \text {, etc. }\end{array}$ \\
\hline oblicuángulo & Geom. Triángulo oblicuángulo. \\
\hline \multicolumn{2}{|l|}{ ochavillo } \\
\hline \multicolumn{2}{|l|}{ odómetro } \\
\hline ordenada & Geom. Línea ordenada. \\
\hline ordenar & $\varnothing$ \\
\hline
\end{tabular}




\begin{tabular}{|c|c|}
\hline ordenatriz & \\
\hline ortogonal & \\
\hline ortogonio & Geom. Triángulo ortogonio. \\
\hline osculación & \\
\hline osculador & \\
\hline $\begin{array}{l}\text { oxígono } \\
\text { /oxigonio }\end{array}$ & Geom. Triángulo oxigonio. \\
\hline pantogonia & \\
\hline paraboloide & \\
\hline paracéntrico & \\
\hline pelecoide & \\
\hline piramidoide & \\
\hline planimetría & $\begin{array}{l}\text { Parte de la topografía, que enseña a representar en una superficie plana } \\
\text { una porción de la terrestre. }\end{array}$ \\
\hline poligonometría & \\
\hline porisma & \\
\hline proyección & $\begin{array}{l}\text { Geom. Representación gráfica o dibujo de una figura sobre los planos } \\
\text { horizontal y vertical, llamados por eso planos de proyección. }\end{array}$ \\
\hline proyectante & \\
\hline punto & $\varnothing$ \\
\hline rabdología & \\
\hline radial & $\varnothing$ \\
\hline rectificar & $\varnothing$ \\
\hline recurrente & $\varnothing$ \\
\hline reducida & \\
\hline regular & $\begin{array}{l}\text { Geom. Dícese de los polígonos y de los poliedros que tienen iguales sus } \\
\text { lados o sus caras y los ángulos que aquellos o estas forman entre sí. }\end{array}$ \\
\hline resecta & \\
\hline residuo & Álg. y Arit. Resta. \\
\hline resta & $\begin{array}{l}\text { Álg. y Arit. Operación de restar, que es una de las cuatro reglas funda- } \\
\text { mentales de la aritmética y álgebra. \| Álg. y Arit. Resultado de la misma } \\
\text { operación. }\end{array}$ \\
\hline resto & Álg. y Arit. Resta. \\
\hline
\end{tabular}




\begin{tabular}{|c|c|}
\hline semejante & $\begin{array}{l}\text { Geom. Dícese de dos figuras distintas sólo por el tamaño y cuyas partes } \\
\text { guardan todas respectivamente la misma proporción. }\end{array}$ \\
\hline signo & $\begin{array}{l}\text { Mat. Señal o figura que se usa en los cálculos, para indicar, ya la natura- } \\
\text { leza de las cantidades, ya las operaciones que se han de ejecutar con } \\
\text { ellas. }\end{array}$ \\
\hline simetría & $\begin{array}{l}\text { Armonía de posición de las partes o puntos similares respecto unos de } \\
\text { otros, y con referencia a punto, línea o cuerpo determinado. }\end{array}$ \\
\hline solidez & Geom. Volumen. \\
\hline sordo & Arit. Raíz sorda. \\
\hline \multicolumn{2}{|l|}{ subcontrarios } \\
\hline submúltiplo & Arit. Aplícase al número contenido en otro varias veces exactamente. \\
\hline \multicolumn{2}{|l|}{ subnormal } \\
\hline \multicolumn{2}{|l|}{ subtangente } \\
\hline \multicolumn{2}{|l|}{ subtriple } \\
\hline \multicolumn{2}{|l|}{ sumatorio } \\
\hline superposición & $\varnothing$ \\
\hline suplemento & $\begin{array}{l}\text { Geom. Ángulo que falta a otro para componer dos rectos. } \| \text { Geom. Arco } \\
\text { de este ángulo, o sea el que falta a otro para completar una semicircun- } \\
\text { ferencia. }\end{array}$ \\
\hline \multicolumn{2}{|l|}{ tautocrona } \\
\hline teodolito & $\begin{array}{l}\text { Mat. Instrumento de precisión que se compone de un círculo horizontal } \\
\text { y un semicírculo vertical, ambos graduados y provistos de anteojos, para } \\
\text { medir ángulos en sus planos respectivos. }\end{array}$ \\
\hline tiralineas & $\begin{array}{l}\text { Instrumento de metal, a modo de pinzas, cuya separación se gradúa con } \\
\text { un tornillo, y sirve para trazar líneas de tinta. }\end{array}$ \\
\hline \multicolumn{2}{|l|}{ tractriz } \\
\hline transcendente & $\varnothing$ \\
\hline transposición & $\varnothing$ \\
\hline traza & $\begin{array}{l}\text { Geom. Descrip. Intersección de una línea o de un plano con los planos } \\
\text { de proyección. }\end{array}$ \\
\hline \multicolumn{2}{|l|}{ triangulación } \\
\hline tridente & $\varnothing$ \\
\hline \multicolumn{2}{|l|}{ triedro } \\
\hline \multicolumn{2}{|l|}{ trilátero } \\
\hline \multicolumn{2}{|l|}{ trocoide } \\
\hline variable & Mat. Cantidad variable. \\
\hline variación & $\varnothing$ \\
\hline
\end{tabular}




\begin{tabular}{|l|l|}
\hline vector & Geom. Radio vector. \\
\hline zetema & $\begin{array}{l}\text { Geom. Parte de la superficie de la esfera, comprendida entre dos planos } \\
\text { paralelos. }\end{array}$ \\
\hline zona
\end{tabular}

Tabla 1. Términos catalogados por Picatoste presentes en el DRAE-1884

Finalmente, otro aspecto destacable es la incorporación ocasional en su catálogo de voces de alguna matización subjetiva o personal. Este hecho se comprueba en los cuarenta términos matemáticos siguientes: ábaco, abscisa, aditivo, algoritmia, ambligonio, cateto, construcción, contorno, conversión, determinado/determinar, discreta, divergente, eje, eminencial, equivalencia, grado, igual, igualdad, incomplejo, irregular, lado, lateral, ley, menos, ortógono, oxígono u oxigonio, proyección, proyectante, punto, dos puntos, rectificar, regular, residuo, resta, resto, semejante y semejanza, simetría, sordo, vector y zona.

En ocasiones se limita a introducir una observación respecto a la voz que generalmente versa sobre su amplio uso por los matemáticos o sobre las acepciones que el diccionario no contempla en la microestructura:

Ábaco. Voz usadísima por los escritores españoles matemáticos y no matemáticos.

Aditivo. No hay tratado de Álgebra en que no se use esta palabra aplicada a los términos positivos.

Construcción. Falta en el Diccionario en su acepción geométrica o gráfica. Eje. Falta la definición de eje de revolución, de simetría, de coordenadas, de una curva, de un cuerpo, etc.; es decir, todas las acepciones geométricas de esta palabra.

Grado. El Diccionario sólo define el grado del círculo. Faltan las definiciones de grado de una potencia, de un término, de una ecuación, de una curva.

También añade, aunque en menor medida, su opinión sobre la necesidad de que el diccionario acoja el término:

Abscisa. Una de las coordenadas necesarias para fijar la posición de un punto en un plano. El diccionario que omite esta palabra, absolutamente necesaria en Geometría analítica, trae abscisión, voz médica [...].

Eminencial. Voz ant. Ecuación exponencial que contiene en sus términos una exponencial función de otra. El diccionario define esta palabra en filosofía.

Menos. Falta la acepción de signo aritmético, que el Diccionario pone en la palabra más; y no hay razón para esta diferencia.

O precisa la inexactitud del trabajo académico con respecto a la falta de lematización de un término que sí se emplea bajo otro artículo lexicográfico. Se contraviene el 
principio de autosuficiencia consistente en no emplear en la definición unidades léxicas que no estén a su vez definidas dentro del diccionario, a fin de evitar las denominadas pistas perdidas (Porto Dapena, 2002: 335):
Amblígono. Lo mismo que ambligonio. El Diccionario no trae esta pala- bra, y sin embargo la usa en la voz triángulo.
Contorno. La suma de las líneas que cierran una figura o polígono. Sin embargo, el Diccionario usa esta palabra en las voces periferia y períme- tro.
Discreta. Cantidad aritmética que crece por grados sensibles. Sin embar- go, el Diccionario usa esta palabra en el artículo Cantidad.
Ortógono. [...] El Diccionario usa, sin embargo, esta palabra en la voz triángulo.
Oxígono u oxigonio. [...] El Diccionario usa, sin embargo, esta palabra en la voz triángulo.
Sordo. Incomensurable. El diccionario usa esta palabra en el artículo raíz.
Vector. Falta esta palabra, que sin embargo el Diccionario usa en el artícu- lo radio.

Por último, la mayoría de los términos de este grupo carece de definición, pese a contar casi todos ellos con alguna información científica o subjetiva: aditivo, algoritmia, cateto, determinar, divergente, eje, equivalencia, grado, igual, igualdad, incomplejo, irregular, lado, lateral, ley, menos, proyección, proyectante, punto, dos puntos, rectificar, regular, residuo, resta, resto, semejante, simetría, vector y zona.

Sin embargo, a estos habría que añadir los términos que se introducen sin estar acompañados de ningún tipo de información ni de definición: equiangular, equidistancia, equidistante, oblicuángulo y trilátero.

\section{CONCLUSIÓN}

El tecnicismo matemático de Felipe Picatoste supone una revisión exhaustiva de la obra lexicográfica de la Academia. En ella, el matemático nos presenta no sólo las voces que no están presentes en el diccionario académico, sino que ofrece un amplio catálogo de términos matemáticos que la Academia debería acoger. Paralelamente, ofrece motivos justificados de por qué estos tecnicismos, al igual que las palabras del lenguaje común que han alcanzado un uso como especializadas, muchos de ellos utilizados desde hace siglos por matemáticos, otros acuñados por el genio inventivo debido a las innovaciones y avances en el terreno de las matemáticas, deben constar en el diccionario de lengua. Para ello analiza las consideraciones y posturas mantenidas por los propios académicos, a la vez que desarrolla unos planteamientos teóricos sobre el lenguaje científico que se acercan a los postulados manejados en la actualidad en los registros de especialidad.

El empobrecimiento del diccionario por la falta de términos matemáticos, que lamenta Picatoste, a diferencia de la situación de otras disciplinas científicas, se vio parcialmente paliado en la siguiente edición del $D R A E$ en la que se incrementó el número de tecnicismos, como hemos tenido ocasión de comprobar. 


\section{REFERENCIAS BIBLIOGRÁFICAS}

Cabré Castellví, María Teresa (1993): La terminología. Teoría, metodología, aplicaciones, Barcelona, Antártida/Empúries.

—_— (2003): «El lenguaje científico desde la terminología», en Bertha Gutiérrez Rodilla, coord., Aproximaciones al lenguaje de la ciencia, Burgos, Instituto Castellano-Leonés de la Lengua, Colección Beltenebros, pp. 19-52.

ECHEGARAY, José (2007): Historia de las matemáticas puras en España, Murcia, Colegio de Ingenieros de Caminos, Canales y Puertos.

ENCICLOPEDIA UNIVERSAL ILUSTRADA EUROPEO-AMERICANA (1975), Madrid, Espasa Calpe.

FERnÁndez SEVILla, Julio (1974): Problemas de lexicografia actual, Bogotá, Publicaciones del Instituto Caro y Cuervo.

GARRIGA ESCRIBANO, Cecilio (2001): «Sobre el diccionario académico: la 12 ed. (1884)», en Antonia María Medina Guerra, coord., Estudios de lexicografía diacrónica del español, Málaga, Universidad de Málaga, pp. 263-315.

GILI GaYA, Samuel (1964): «El lenguaje de la ciencia y de la técnica», en Presente y futuro de la lengua española, Madrid, Ediciones Cultura Hispánica, pp. 269-276.

GutiÉRrez RoDilla, Bertha (1998): La ciencia empieza en la palabra. Análisis e historia del lenguaje cientifico, Barcelona, Ediciones Península.

PICATOSTE Y RodRÍGUEZ, Felipe (1873): El tecnicismo matemático en el Diccionario de la Academia Española, Madrid, Segundo Martínez.

PORTO DAPEnA, José Álvaro (2002): Manual de técnica lexicográfica, Madrid, Arco/Libros.

REAL ACADEMia ESPAÑola $\left(1869^{11}\right)$ : Diccionario de la lengua castellana, Madrid, Manuel Rivadeneyra.

- $\left(1884^{12}\right)$ : Diccionario de la lengua castellana, Madrid, Gregorio Hernando.

ZAMORA ViCEnTE, Alonso (1999): Historia de la Real Academia Española, Madrid, Espasa Calpe. 